\title{
28. DATA REPORT: PHASE CHEMISTRY OF VOLCANIC ASH FROM SITE 9491
}

\author{
Peter A. Cawood ${ }^{2}$ and Evan C. Leitch ${ }^{3}$
}

\section{INTRODUCTION}

In this paper we report compositional data for a number of ash layers recovered at Ocean Drilling Program (ODP) Site 949 (Fig. 1). We have selected eight samples ranging in age from early Miocene to early Pleistocene (Table 1) that provide a reconnaissance of ash compositions and of the nature of preserved important pyrogenic phases. Previous investigations of marine tephra deposits adjacent to the Lesser Antilles arc have been mostly concerned with late Quaternary deposits (e.g. Sigurdsson et al., 1980; Carey and Sigurdsson, 1980). The data presented here thus extend the stratigraphic record considerably. The new data compliment the compilation of Natland (1984), who summarized the distribution of volcanic ash-fall layers in cores from Deep Sea Drilling Project (DSDP) Sites 541, 542, and 543, showing that ash was concentrated in sediments of middle Miocene, late Miocene, early Miocene, and Pleistocene-Holocene age. Because of the limited intervals over which coring was attempted on Leg 156 and low core recovery, our sampling has been incomplete. We have, however, analyzed phases from each of the major eruptive pulses identified by Natland (1984), who did not present any analytical data.

\section{SAMPLES}

Ash concentrations recovered at Site 949 are characterized by a coarser grain size and darker color than the surrounding claystones. Most ash is of coarse silt grade, but scattered sand-sized grains are present. The ash ranges in color from light gray to almost black, with some having a greenish hue. Crystal fragments make up between about $1 \%$ and $30 \%$ of the total volcanic debris, but most samples contain between $5 \%$ and $15 \%$, consistent with a primary fall origin for the ash layers (Natland, 1984). Layers of mass-flow origin tend to be either depleted $(<1 \mathrm{vol} \%)$ or enriched $(30-50 \mathrm{vol} \%)$ in phenocrysts (Bednarz and Schmincke, 1994). Although turbidite emplacement might account for the abundant phenocrysts $(\sim 30 \%)$ in Sample 156$949-1 \mathrm{H}-1,75-77 \mathrm{~cm}$, it is unlikely because the drill site is protected from the influx of mass-flows from the Lesser Antilles arc by the Barbados Ridge (Natland, 1984).

Undisturbed ash layers, which range in thickness up to $5 \mathrm{~cm}$, have sharp bases and tops and may be internally laminated. Much ash, however, has been disturbed subsequent to deposition and intermixed with the adjacent claystone to varying degrees, mainly by burrowing organisms. Mild disturbance is marked by the bioturbation of the upper part of ash layers and the production of a highly irregular upper contact. Commonly, the whole layer has been bioturbated and both top and bottom of the bed are irregular. More intense bioturbation activity has resulted in the dispersion of the ash in small blebs through-

${ }^{1}$ Shipley, T.H., Ogawa, Y., Blum, P., and Bahr, J.M. (Eds.), 1997. Proc. ODP, Sci. Results, 156: College Station, TX (Ocean Drilling Program).

${ }^{2}$ School of Applied Geology, Curtin University of Technology, GPO Box U1987, Perth. WA 6001. Australia.

${ }^{3}$ Department of Applied Geology, University of Technology, Sydney, PO Box 123, Broadway, NSW 2007, Australia. e.leitch@uts.edu.au out adjacent claystone, and, in its extreme manifestation, has given rise to ashy claystone in which individual ash grains, or aggregates of a few grains, are dispersed through highly bioturbated claystone. Zones of dispersed ash range up to $40 \mathrm{~cm}$ in thickness.

Information concerning the eight samples for which we have obtained analytical data is summarized in Table 1. All samples are from Site 949, drilled into the accretionary subduction complex of the northern Barbados Ridge, 2-km west of the deformation front and about 240-km east-northeast of Martinique (Shipley, Ogawa, Blum, et al., 1995). Site 949 is located on the northwest tip of the Tiburon Rise, in the same area as was investigated during DSDP Leg 78A (Sites 541-543) and ODP Leg 110 (Sites 671-676; Fig. 1).

The age of the samples has been established from biostratigraphic data derived from closely associated rocks. Uncertainty concerning the age of Samples 156-949B-3X-1, 56-57 cm, and 156-949B-3X-5, $53-54 \mathrm{~cm}$, derives from the presence of a stratigraphic inversion between Cores 156-949B-2X and 7X. It is likely that the responsible fault occurs in Core 156-949B-6X, and hence the samples are probably late middle Miocene (Shipley, Ogawa, Blum, et al., 1995).

\section{PETROGRAPHY}

Plagioclase is the most abundant phenocryst mineral and is present in all samples. Other pyrogenic minerals include quartz, hornblende, orthopyroxene, clinopyroxene, opaque oxide minerals, and potassium feldspar. Quartz was observed in all samples except $156-949 \mathrm{~A}-1 \mathrm{H}-1,75-77 \mathrm{~cm}$, and is relatively common in 156-949B$3 \mathrm{X}-1,56-57 \mathrm{~cm}$, and $156-949 \mathrm{~B}-3 \mathrm{X}-5,53-54 \mathrm{~cm}$. The presence of quartz phenocrysts suggests some silicic volcanic activity at the time of deposition of the ash samples. Potassium feldspar is restricted to Samples 156-949B-19X-4, 10-11 cm, and 156-949B-7X-2, 63-64 $\mathrm{cm}$. Hornblende and pyroxene occur in Samples 156-949A-1H-1, 35-37 cm, 156-949A-1H-1, 75-77 cm, 156-949A-1H-CC, 5-7 cm, and 156-949B-19X-4, 10-11 cm. Neither olivine nor biotite were observed in any sample.

Ubiquitous volcanic glass ranges from optically unaltered colorless shards, indicative of a high silica content, to highly altered devitrified clays, the latter widespread in Samples 156-949B-3X-1, 56-57 $\mathrm{cm}, 156-949 \mathrm{~B}-3 \mathrm{X}-5,53-54 \mathrm{~cm}, 156-949 \mathrm{~B}-7 \mathrm{X}-1,141-142 \mathrm{~cm}$, and $156-949 \mathrm{~B}-7 \mathrm{X}-2,63-64 \mathrm{~cm}$. Shards are mainly of cuspate and pumice types (Fisher and Schmincke, 1984).

\section{PHASE CHEMISTRY}

Mineral and glass analyses were carried out at Macquarie University, NSW, Australia, using a Cameca SX50 electron microprobe fitted with five wavelength dispersive spectrometers. Operating conditions for mineral analyses were an accelerating voltage of $15 \mathrm{KV}$, beam current of 20 nanoamps (measured in Faraday cup), beam diameter of $2 \mu \mathrm{m}$, and count times of $20 \mathrm{~s}$. To minimize alkali loss for volcanic glass analyses (Nielsen and Sigurdsson, 1981), the microprobe beam was defocussed to $10 \mu \mathrm{m}$, beam current reduced to 6 nanoamps, $\mathrm{K}$ and $\mathrm{Na}$ were analyzed first on their respective spec- 


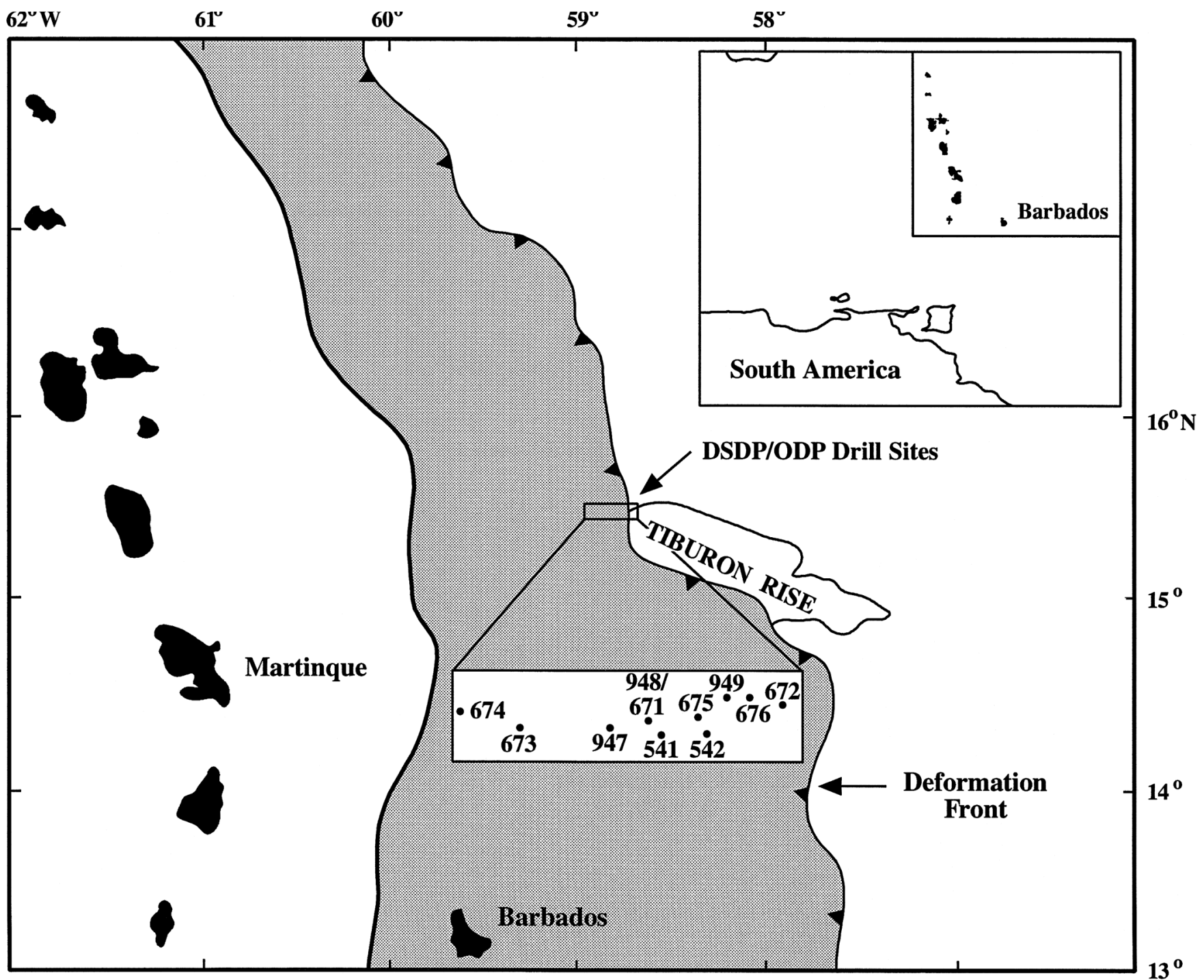

Figure 1. Location of deep-sea drilling area, north Barbados Ridge. The stippled zone outlines the extent of the accretionary subduction complex.

Table 1. Ash samples analyzed, Site 949.

\begin{tabular}{|c|c|c|}
\hline $\begin{array}{l}\text { Core, section, } \\
\text { interval }(\mathrm{cm})\end{array}$ & Age & Sample character \\
\hline $\begin{array}{l}156-949 \mathrm{~A}- \\
1 \mathrm{H}-1,35-37 \\
1 \mathrm{H}-1,75-77 \\
1 \mathrm{H}-\mathrm{CC}, 5-7\end{array}$ & $\begin{array}{l}\text { early Pleistocene } \\
\text { early Pleistocene } \\
\text { early Pleistocene }\end{array}$ & $\begin{array}{l}\text { Disrupted layer } \\
\text { Disrupted layer } \\
\text { Disrupted layer }\end{array}$ \\
\hline $\begin{array}{l}\text { 156-949B- } \\
\text { 3X-1, 56-57 } \\
3 \mathrm{X}-5,53-54 \\
7 \mathrm{X}-1,141-142 \\
7 \mathrm{X}-2,63-64 \\
19 \mathrm{X}-4,10-11\end{array}$ & $\begin{array}{l}\text { ? late middle Miocene } \\
\text { ? late middle Miocene } \\
\text { late Miocene } \\
\text { late Miocene } \\
\text { early Miocene }\end{array}$ & $\begin{array}{l}\text { Dispersed ash } \\
\text { Dispersed ash } \\
\text { Discrete layer } \\
\text { Discrete layer } \\
\text { Disrupted layer }\end{array}$ \\
\hline
\end{tabular}

trometers, and the peak was measured before the two background measurements. All analyses were corrected using the online correction procedures outlined by Pouchou and Pichoir (1984).

Some 550 analyses of feldspar, pyroxene, amphibole, opaque oxide minerals, and volcanic glass were carried out on the eight ash samples. Analyses were mostly restricted to the core region of grains as the fragmental nature of the rocks and the broken nature of many grains prevented accurate determination of original grain rims. Rep-
Table 2. Plagioclase compositional ranges in ash samples, Site 949.

\begin{tabular}{cc}
\hline $\begin{array}{c}\text { Core, section, } \\
\text { interval }(\mathrm{cm})\end{array}$ & $\begin{array}{c}\text { Plagioclase } \\
\text { compositional range }\end{array}$ \\
\hline $156-949 \mathrm{~A}-$ & \\
$1 \mathrm{H}-1,35-37$ & $\mathrm{An}_{82}-\mathrm{An}_{32}$ \\
$1 \mathrm{H}-1,75-77$ & $\mathrm{An}_{83}-\mathrm{An}_{47}$ \\
$1 \mathrm{H}-\mathrm{CC}, 5-7$ & $\mathrm{An}_{90}-\mathrm{An}_{22}$ \\
$156-949 \mathrm{~B}-$ & \\
$3 \mathrm{X}-1,56-57$ & \\
$3 \mathrm{X}-5,53-54$ & $\mathrm{An}_{63}-\mathrm{An}_{7}$ \\
$7 \mathrm{X}-1,141-142$ & $\mathrm{An}_{53}-\mathrm{An}_{0}$ \\
$7 \mathrm{X}-2,63-64$ & $\mathrm{An}_{78}-\mathrm{An}_{7}$ \\
$19 \mathrm{X}-4,10-11$ & $\mathrm{An}_{73}-\mathrm{An}_{0}$ \\
& $\mathrm{An}_{85}-\mathrm{An}_{10}$ \\
\hline
\end{tabular}

resentative analyses are presented in Tables $2-6$ and plotted in Figures 2-8.

\section{Feldspar}

Feldspar is largely of plagioclase composition, but potassium feldspar is a minor component of Samples 156-949B-19X-4, 10-11 $\mathrm{cm}$, and 156-949B-7X-2, 63-64 cm (Tables 2, 3). The compositional 
Table 3. Selected microprobe analyses of feldspar grains from Site 949 volcanic ash samples.

\begin{tabular}{|c|c|c|c|c|c|c|c|c|c|c|}
\hline Core, section: & 949B-3X-5 & 949B-3X-1 & $949 B-3 X-5$ & 949B-19X-4 & 949A-1H-CC & 949B-7X-2 & 949B-7X-2 & 949A-1H-CC & 949B-7X-2 & 949A-1H-CC \\
\hline Interval $(\mathrm{cm})$ : & $53-54$ & $56-57$ & $53-54$ & $10-11$ & $5-7$ & $63-64$ & $63-64$ & $5-7$ & $63-64$ & $5-7$ \\
\hline Analysis: & 1 & 2 & 3 & 4 & 5 & 6 & 7 & 8 & 9 & 10 \\
\hline $\mathrm{SiO}_{2}$ & 69.13 & 61.96 & 58.12 & 56.38 & 55.18 & 54.64 & 53.97 & 53.65 & 53.80 & 53.34 \\
\hline $\mathrm{Al}_{2} \mathrm{O}_{3}$ & 19.55 & 23.91 & 24.97 & 25.98 & 27.17 & 27.93 & 28.51 & 28.22 & 28.26 & 28.45 \\
\hline $\mathrm{FeO}$ & 0.17 & 0.24 & 0.79 & 0.71 & 0.42 & 0.53 & 0.45 & 0.50 & 0.66 & 0.31 \\
\hline $\mathrm{CaO}$ & 0.00 & 5.68 & 8.11 & 9.45 & 10.64 & 11.05 & 11.53 & 11.76 & 11.88 & 12.04 \\
\hline $\mathrm{Na}_{2} \mathrm{O}$ & 11.56 & 8.01 & 6.67 & 5.97 & 5.60 & 5.21 & 5.18 & 4.78 & 4.82 & 4.72 \\
\hline $\mathrm{K}_{2} \mathrm{O}$ & 0.03 & 0.64 & 0.44 & 0.39 & 0.17 & 0.12 & 0.08 & 0.25 & 0.08 & 0.10 \\
\hline Total & 100.45 & 100.44 & 99.10 & 98.88 & 99.17 & 99.48 & 99.72 & 99.16 & 99.50 & 98.96 \\
\hline Core, section: & 949B-7X-1 & 949B-3X-1 & 949B-7X-1 & 949A-1H-1 & 949A-1H-1 & 949B-19X-4 & 949A-1H-1 & 949B-19X-4 & $949 B-7 X-2$ & 949B-19X-4 \\
\hline Interval $(\mathrm{cm})$ : & $141-142$ & $56-57$ & $141-142$ & $35-37$ & $75-77$ & $10-11$ & $75-77$ & $10-11$ & $63-64$ & $10-11$ \\
\hline Analysis: & 11 & 12 & 13 & 14 & 15 & 16 & 17 & 18 & 19 & 20 \\
\hline $\mathrm{SiO}_{2}$ & 53.08 & 52.22 & 51.06 & 50.94 & 48.98 & 48.72 & 48.60 & 46.50 & 64.16 & 65.86 \\
\hline $\mathrm{Al}_{2} \mathrm{O}_{3}$ & 28.52 & 29.44 & 30.10 & 30.68 & 31.81 & 31.38 & 32.40 & 33.10 & 20.90 & 19.25 \\
\hline $\mathrm{FeO}$ & 0.73 & 0.53 & 0.51 & 0.36 & 0.45 & 0.54 & 0.36 & 0.55 & 0.39 & 0.24 \\
\hline $\mathrm{CaO}$ & 12.33 & 12.83 & 13.71 & 14.35 & 15.73 & 16.28 & 16.36 & 17.15 & 2.82 & 0.97 \\
\hline $\mathrm{Na}_{2} \mathrm{O}$ & 4.54 & 4.03 & 3.74 & 3.46 & 2.58 & 2.52 & 2.20 & 1.85 & 8.21 & 5.53 \\
\hline $\mathrm{K}_{2} \mathrm{O}$ & 0.24 & 0.17 & 0.08 & 0.07 & 0.09 & 0.03 & 0.07 & 0.01 & 2.14 & 7.98 \\
\hline Total & 99.44 & 99.21 & 99.20 & 99.87 & 99.64 & 99.47 & 99.99 & 99.15 & 98.62 & 99.83 \\
\hline
\end{tabular}

Note: Analyses 1-18 are of plagioclase grains and analyses 19 and 20 are of potassium feldspar grains.

Table 4. Selected microprobe analyses of pyroxene grains from Hole 949A volcanic ash samples.

\begin{tabular}{|c|c|c|c|c|c|c|c|c|c|c|}
\hline Core, section: & $1 \mathrm{H}-1$ & $1 \mathrm{H}-1$ & $1 \mathrm{H}-1$ & $1 \mathrm{H}-1$ & $1 \mathrm{H}-1$ & $1 \mathrm{H}-1$ & $1 \mathrm{H}-1$ & $1 \mathrm{H}-1$ & $1 \mathrm{H}-1$ & $1 \mathrm{H}-\mathrm{CC}$ \\
\hline Interval $(\mathrm{cm})$ : & $35-37$ & $35-37$ & $35-37$ & $35-37$ & $75-77$ & $75-77$ & $75-77$ & $75-77$ & $75-77$ & $5-7$ \\
\hline Analysis: & 1 & 2 & 3 & 4 & 5 & 6 & 7 & 8 & 9 & 10 \\
\hline $\mathrm{SiO}_{2}$ & 51.67 & 52.17 & 51.35 & 50.98 & 50.75 & 51.08 & 50.95 & 51.35 & 51.45 & 51.74 \\
\hline $\mathrm{TiO}_{2}^{2}$ & 0.16 & 0.09 & 0.15 & 0.15 & 0.10 & 0.23 & 0.14 & 0.14 & 0.13 & 0.14 \\
\hline $\mathrm{Al}_{2} \mathrm{O}_{3}$ & 0.57 & 0.53 & 0.80 & 0.51 & 0.47 & 1.65 & 0.95 & 1.00 & 0.61 & 1.09 \\
\hline $\mathrm{FeO}$ & 27.75 & 26.81 & 27.08 & 26.90 & 30.50 & 26.75 & 28.97 & 28.75 & 28.90 & 23.78 \\
\hline $\mathrm{MnO}$ & 1.00 & 1.15 & 0.96 & 1.11 & 1.36 & 0.85 & 1.44 & 1.16 & 1.37 & 1.36 \\
\hline $\mathrm{MgO}$ & 18.28 & 18.86 & 18.31 & 18.55 & 16.19 & 18.48 & 16.92 & 17.31 & 17.38 & 21.15 \\
\hline $\mathrm{CaO}$ & 1.08 & 1.04 & 1.20 & 1.04 & 0.94 & 1.44 & 0.89 & 0.85 & 0.85 & 0.68 \\
\hline $\mathrm{Na}_{2} \mathrm{O}$ & 0.01 & 0.06 & 0.02 & 0.05 & 0.02 & 0.02 & 0.04 & 0.02 & 0.01 & 0.00 \\
\hline $\mathrm{K}_{2} \mathrm{O}$ & 0.01 & 0.03 & 0.02 & 0.01 & 0.02 & 0.01 & 0.00 & 0.01 & 0.00 & 0.01 \\
\hline Total & 100.53 & 100.73 & 99.90 & 99.30 & 100.33 & 100.51 & 100.30 & 100.59 & 100.71 & 99.94 \\
\hline Core, section: & 1H-CC & 1H-CC & $1 \mathrm{H}-\mathrm{CC}$ & $1 \mathrm{H}-\mathrm{CC}$ & $1 \mathrm{H}-\mathrm{CC}$ & $1 \mathrm{H}-1$ & $1 \mathrm{H}-1$ & $1 \mathrm{H}-1$ & $1 \mathrm{H}-\mathrm{CC}$ & $1 \mathrm{H}-\mathrm{CC}$ \\
\hline Interval $(\mathrm{cm})$ : & $5-7$ & $5-7$ & $5-7$ & $5-7$ & $5-7$ & $35-37$ & $35-37$ & $75-77$ & $5-7$ & $5-7$ \\
\hline Analysis: & 11 & 12 & 13 & 14 & 15 & 16 & 17 & 18 & 19 & 20 \\
\hline $\mathrm{SiO}_{2}$ & 53.21 & 52.70 & 54.44 & 50.82 & 52.34 & 52.16 & 51.74 & 52.07 & 50.25 & 51.70 \\
\hline $\mathrm{TiO}_{2}$ & 0.13 & 0.13 & 0.15 & 0.20 & 0.13 & 0.22 & 0.16 & 0.24 & 0.51 & 0.36 \\
\hline $\mathrm{Al}_{2} \mathrm{O}_{3}$ & 0.82 & 0.64 & 0.71 & 3.31 & 1.14 & 1.09 & 1.01 & 1.14 & 3.29 & 1.68 \\
\hline $\mathrm{FeO}$ & 18.98 & 22.95 & 14.52 & 18.84 & 21.54 & 11.63 & 11.97 & 11.70 & 10.31 & 10.22 \\
\hline $\mathrm{MnO}$ & 0.60 & 1.17 & 0.66 & 0.73 & 1.06 & 0.54 & 0.49 & 0.49 & 0.44 & 0.55 \\
\hline $\mathrm{MgO}$ & 24.45 & 21.61 & 26.87 & 23.76 & 22.32 & 13.00 & 12.74 & 13.23 & 13.54 & 14.01 \\
\hline $\mathrm{CaO}$ & 1.58 & 1.06 & 1.32 & 1.58 & 1.61 & 21.19 & 21.19 & 20.90 & 20.98 & 21.44 \\
\hline $\mathrm{Na}_{2} \mathrm{O}$ & 0.01 & 0.05 & 0.15 & 0.02 & 0.01 & 0.25 & 0.25 & 0.29 & 0.24 & 0.26 \\
\hline $\mathrm{K}_{2} \mathrm{O}$ & 0.01 & 0.00 & 0.03 & 0.00 & 0.03 & 0.00 & 0.00 & 0.00 & 0.00 & 0.02 \\
\hline Total & 99.80 & 100.30 & 98.86 & 99.27 & 100.19 & 100.07 & 99.55 & 100.06 & 99.55 & 100.25 \\
\hline
\end{tabular}

Note: Analyses 1-15 are of orthopyroxene grains and analyses 16-20 are of clinopyroxene grains.

range of plagioclase analyses from all samples extends from $\mathrm{An}_{90}$ to $\mathrm{An}_{0}$, but with a more restricted range for individual samples (Fig. 2; Table 2). The content of Or increases with increasing Ab. The majority of the plagioclase analyses $(61 \%)$ are labradorite and over $97 \%$ of analyses fall in the andesine to bytownite range (Fig. 3). Albite and oligoclase constitute less than $3 \%$ of analyzed grains. Albite is restricted to Samples 156-949B-3X-1, 56-57 cm, 156-949B-3X-5, 53$57 \mathrm{~cm}$, and 156-949B-7X-1, 141-142 cm, where it is accompanied by altered glass, indicating it is probably of diagenetic origin.

$\mathrm{FeO}$ is a minor component in volcanic plagioclase (Gill, 1981). Contents of up to $1.2 \%$ were measured in the sample set, but the composition in Samples 156-949A-1H-1, 35-37 cm, 156-949A-1H-1, $75-77 \mathrm{~cm}$, and $156-949 \mathrm{~A}-1 \mathrm{H}-\mathrm{CC}, 5-7 \mathrm{~cm}$, ranges between $0.2 \%$ and $0.6 \%$. Sample 156-949B-7X-1, 141-142 cm, has values between $0.2 \%$ and $0.8 \%$, and Samples 156-949B-19X-4, 10-11 cm, 156949B-3X-1, 56-57 cm, 156-949B-3X-5, 53-54 cm, and 156-949B$7 \mathrm{X}-2,63-64 \mathrm{~cm}$, have contents up to $1.2 \%$, although most analyses in this last group fall in the $0.4 \%-1.0 \%$ range. In all samples, $\mathrm{FeO}$ content is independent of An composition for contents greater than around $0.2 \% \mathrm{FeO}$. Albite and oligoclase mostly have $\mathrm{FeO}$ less than
$0.25 \%$, although in Sample 156-949A-1H-CC, 5-7 cm, oligoclase has an $\mathrm{FeO}$ content of $1.2 \%$.

Potassium feldspar occurs in Samples 156-949B-19X-4, 10-11 $\mathrm{cm}$, and 156-949B-7X-2, 63-64 cm (Fig. 2), and both samples include grains of anorthoclase and sanidine. Sanidine compositions in the two samples are similar with Ab:Or around 50:50 and An contents of $<5 \mathrm{~mol} \%$. The $\mathrm{FeO}$ content of the analyzed potassium feldspar grains is low $(<0.4 \%)$. In Sample 156-949B-19X-4, 10-11 cm, potassium feldspar is accompanied by abundant low-K silicic glass, suggesting a mixed source for this ash.

\section{Pyroxene}

Pyroxene occurs in Samples 156-949A-1H-1, 35-37 cm, 156949A-1H-1, 75-77 cm, and 156-949A-1H-CC, 5-7 cm, and includes orthopyroxene and scarce calcic clinopyroxene (Table 4). On the pyroxene quadrilateral plot, clinopyroxene analyses from all samples cluster in the augite field close to the augite/salite boundary at around $\mathrm{Ca}_{44} \mathrm{Mg}_{37} \mathrm{Fe}_{19}$ (Fig. 4). The overall high $\mathrm{Ca}$ and moderate Fe values of the analyzed clinopyroxene grains are similar to those from magmat- 
Table 5. Selected microprobe analyses of amphibole grains from Hole 949A volcanic ash samples.

\begin{tabular}{|c|c|c|c|c|c|c|c|c|c|c|c|}
\hline Core, section: & $1 \mathrm{H}-1$ & $1 \mathrm{H}-1$ & $1 \mathrm{H}-1$ & $1 \mathrm{H}-1$ & $1 \mathrm{H}-1$ & $1 \mathrm{H}-1$ & $1 \mathrm{H}-1$ & $1 \mathrm{H}-1$ & $1 \mathrm{H}-\mathrm{CC}$ & $1 \mathrm{H}-\mathrm{CC}$ & $1 \mathrm{H}-\mathrm{CC}$ \\
\hline Interval $(\mathrm{cm})$ : & $75-77$ & $75-77$ & $75-77$ & $75-77$ & $75-77$ & $75-77$ & $75-77$ & $75-77$ & $5-7$ & $5-7$ & $5-7$ \\
\hline Analysis: & 1 & 2 & 3 & 4 & 5 & 6 & 7 & 8 & 9 & 10 & 11 \\
\hline $\mathrm{SiO}_{2}$ & 47.73 & 46.06 & 47.45 & 46.69 & 47.46 & 47.97 & 45.86 & 45.91 & 47.60 & 47.14 & 47.83 \\
\hline $\mathrm{TiO}_{2}$ & 1.42 & 1.71 & 1.35 & 1.59 & 1.41 & 1.31 & 1.63 & 1.14 & 1.06 & 1.33 & 1.10 \\
\hline $\mathrm{Al}_{2} \mathrm{O}_{3}$ & 7.08 & 8.14 & 7.06 & 7.37 & 6.97 & 6.62 & 7.62 & 8.54 & 6.81 & 7.06 & 6.89 \\
\hline $\mathrm{FeO}$ & 16.68 & 17.68 & 18.07 & 17.01 & 16.38 & 17.62 & 17.47 & 14.83 & 14.86 & 14.29 & 14.84 \\
\hline $\mathrm{MnO}$ & 0.35 & 0.40 & 0.49 & 0.41 & 0.34 & 0.42 & 0.37 & 0.52 & 0.74 & 0.41 & 0.56 \\
\hline $\mathrm{MgO}$ & 12.73 & 11.89 & 11.85 & 12.26 & 12.89 & 12.40 & 11.59 & 13.90 & 14.06 & 13.83 & 14.20 \\
\hline $\mathrm{CaO}$ & 11.03 & 10.90 & 10.37 & 11.03 & 11.09 & 10.81 & 11.00 & 9.82 & 10.20 & 10.88 & 10.33 \\
\hline $\mathrm{Na}_{2} \mathrm{O}$ & 1.18 & 1.37 & 1.18 & 1.27 & 1.24 & 1.19 & 1.32 & 1.41 & 1.08 & 1.16 & 1.12 \\
\hline $\mathrm{K}_{2} \mathrm{O}$ & 0.26 & 0.44 & 0.33 & 0.36 & 0.24 & 0.35 & 0.43 & 0.19 & 0.16 & 0.21 & 0.22 \\
\hline Total & 98.47 & 98.57 & 98.16 & 97.99 & 98.02 & 98.69 & 97.28 & 96.27 & 96.59 & 96.31 & 97.10 \\
\hline
\end{tabular}

Table 6. Selected microprobe analyses of glass from Site 949 volcanic ash samples.

\begin{tabular}{|c|c|c|c|c|c|c|c|c|c|c|}
\hline Core, section: & $949 \mathrm{~B}-19 \mathrm{H}-4$ & 949B-19H-4 & 949B-19H-4 & 949B-19H-4 & 949B-19H-4 & 949B-19H-4 & 949B-19H-4 & 949A-1H-CC & 949A-1H-CC & 949A-1HCC \\
\hline Interval $(\mathrm{cm})$ : & $10-11$ & $10-11$ & $10-11$ & $10-11$ & $10-11$ & $10-11$ & $10-11$ & $5-7$ & $5-7$ & $5-7$ \\
\hline Analysis: & 1 & 2 & 3 & 4 & 5 & 6 & 7 & 8 & 9 & 10 \\
\hline $\mathrm{SiO}_{2}$ & 65.17 & 66.81 & 69.38 & 69.65 & 70.33 & 70.64 & 71.05 & 69.60 & 70.42 & 71.05 \\
\hline $\mathrm{TiO}_{2}$ & 0.59 & $\begin{array}{r}0.52 \\
0.51\end{array}$ & 0.33 & 0.36 & 0.34 & 0.41 & 0.29 & 0.02 & 0.08 & 0.33 \\
\hline $\mathrm{Al}_{2} \mathrm{O}_{3}$ & 12.96 & 12.51 & 12.06 & 12.27 & 12.22 & 11.79 & 12.05 & 17.33 & 15.97 & 11.17 \\
\hline $\mathrm{FeO}$ & 5.07 & 4.59 & 3.73 & 3.73 & 3.57 & 3.97 & 3.65 & 1.02 & 0.81 & 3.23 \\
\hline $\mathrm{MnO}$ & 0.11 & 0.11 & 0.04 & 0.20 & 0.11 & 0.04 & 0.05 & 0.08 & 0.11 & 0.20 \\
\hline $\mathrm{MgO}$ & 0.72 & 0.65 & 0.28 & 0.30 & 0.25 & 0.27 & 0.30 & 0.14 & 0.14 & 1.54 \\
\hline $\mathrm{CaO}$ & 3.59 & 3.14 & 2.53 & 2.47 & 2.31 & 2.35 & 2.35 & 4.31 & 3.90 & 3.16 \\
\hline $\mathrm{Na}_{2} \mathrm{O}$ & 3.22 & 3.84 & 4.12 & 4.34 & 4.16 & 3.76 & 4.18 & 4.24 & 4.33 & 3.26 \\
\hline $\mathrm{K}_{2} \mathrm{O}$ & 0.77 & 0.77 & 0.90 & 0.90 & 0.93 & 0.93 & 0.83 & 1.55 & 1.65 & 2.09 \\
\hline $\mathrm{P}_{2} \mathrm{O}_{5}$ & 0.16 & 0.16 & 0.00 & 0.00 & 0.00 & 0.09 & 0.00 & 0.00 & 0.10 & 0.00 \\
\hline Total & 92.35 & 93.09 & 93.36 & 94.22 & 94.21 & 94.25 & 94.74 & 98.30 & 97.51 & 96.04 \\
\hline Core, section: & 949A-1H-CC & 949A-1H-CC & 949A-1H-CC & 949A-1H-CC & 949A-1H-CC & 949A-1H-CC & 949A-1H-1 & $949 \mathrm{~A}-1 \mathrm{H}-1$ & 949A-1H-1 & 949A-1H-1 \\
\hline Interval $(\mathrm{cm})$ : & $5-7$ & $5-7$ & $5-7$ & $5-7$ & $5-7$ & $5-7$ & $75-77$ & $75-77$ & $75-77$ & $75-77$ \\
\hline Analysis: & 11 & 12 & 13 & 14 & 15 & 16 & 17 & 18 & 19 & 20 \\
\hline $\mathrm{SiO}_{2}$ & 71.14 & 71.31 & 72.80 & 73.20 & 73.70 & 74.05 & 67.76 & 71.61 & 73.29 & 73.88 \\
\hline $\mathrm{TiO}_{2}$ & 0.33 & 0.20 & 0.20 & 0.25 & 0.14 & 0.15 & 0.22 & $\begin{array}{r}11.01 \\
0.24\end{array}$ & $\begin{array}{r}75.29 \\
0.20\end{array}$ & $\begin{array}{r}15.00 \\
0.11\end{array}$ \\
\hline $\mathrm{Al}_{2} \mathrm{O}_{3}$ & 16.17 & 10.86 & 12.50 & 12.51 & 11.98 & 11.95 & 16.81 & 11.12 & 12.36 & 11.55 \\
\hline $\mathrm{FeO}$ & 1.16 & 1.22 & 1.48 & 2.03 & 1.05 & 1.46 & 1.25 & 1.73 & 1.72 & 1.61 \\
\hline $\mathrm{MnO}$ & 0.00 & 0.01 & 0.12 & 0.08 & 0.00 & 0.17 & 0.02 & 0.10 & 0.00 & 0.02 \\
\hline $\mathrm{MgO}$ & 0.03 & 0.28 & 0.33 & 0.30 & 0.25 & 0.21 & 0.15 & 0.22 & 0.27 & 0.23 \\
\hline $\mathrm{CaO}$ & 3.95 & 1.60 & 1.81 & 2.55 & 1.82 & 1.53 & 4.80 & 1.71 & 2.16 & 1.75 \\
\hline $\mathrm{Na}_{2} \mathrm{O}$ & 3.98 & 3.08 & 3.87 & 3.78 & 3.70 & 3.54 & 3.73 & 3.20 & 2.27 & 3.23 \\
\hline $\mathrm{K}_{2} \mathrm{O}$ & 1.22 & 2.59 & 2.03 & 2.01 & 2.10 & 2.44 & 1.74 & 2.74 & 2.47 & 2.52 \\
\hline $\mathrm{P}_{2} \mathrm{O}_{5}$ & 0.00 & 0.00 & 0.00 & 0.00 & 0.07 & 0.00 & 0.00 & 0.00 & 0.00 & 0.00 \\
\hline Total & 97.98 & 91.15 & 95.14 & 96.72 & 94.82 & 95.50 & 96.48 & 92.67 & 94.75 & 94.91 \\
\hline Core, section: & 949A-1H-1 & 949A-1H-1 & 949A-1H-1 & $949 \mathrm{~A}-1 \mathrm{H}-1$ & 949A-1H-1 & 949A-1H-1 & $949 \mathrm{~A}-1 \mathrm{H}-1$ & $949 \mathrm{~A}-1 \mathrm{H}-1$ & 949A-1H-1 & 949A-1H-1 \\
\hline Interval $(\mathrm{cm})$ : & $75-77$ & $75-77$ & $75-77$ & $75-77$ & $35-37$ & $35-37$ & $35-37$ & $35-37$ & $35-37$ & $35-37$ \\
\hline Analysis: & 21 & 22 & 23 & 24 & 25 & 26 & 27 & 28 & 29 & 30 \\
\hline $\mathrm{SiO}_{2}$ & 74.27 & 74.57 & 75.30 & 77.56 & 70.96 & 73.19 & 73.72 & 74.39 & 74.87 & 75.08 \\
\hline $\begin{array}{l}\mathrm{SiO}_{2} \\
\mathrm{TiO}_{2}\end{array}$ & 0.15 & 0.15 & 0.24 & 0.15 & $\begin{array}{r}0.17 \\
0.17\end{array}$ & 0.24 & 0.28 & 0.23 & 0.14 & $\begin{array}{r}0.00 \\
0.25\end{array}$ \\
\hline $\mathrm{Al}_{2} \mathrm{O}_{3}$ & 11.76 & 11.57 & 12.41 & 11.99 & 11.34 & 11.93 & 12.00 & 11.40 & 11.56 & 11.56 \\
\hline $\begin{array}{l}\mathrm{Al}_{2} \mathrm{O}_{3} \\
\mathrm{FeO}\end{array}$ & $\begin{array}{r}11 . / 0 \\
1.23\end{array}$ & $\begin{array}{r}11.3 / \\
1.55\end{array}$ & $\begin{array}{r}12.41 \\
1.86\end{array}$ & $\begin{array}{r}11.99 \\
1.43\end{array}$ & $\begin{array}{r}11.34 \\
1.54\end{array}$ & $\begin{array}{r}11.93 \\
1.54\end{array}$ & $\begin{array}{r}12.00 \\
1.79\end{array}$ & $\begin{array}{r}11.40 \\
1.60\end{array}$ & $\begin{array}{r}11.50 \\
1.60\end{array}$ & $\begin{array}{r}11.50 \\
1.63\end{array}$ \\
\hline $\mathrm{MnO}$ & 0.08 & 0.00 & 0.05 & 0.07 & 0.14 & 0.00 & 0.11 & 0.03 & 0.00 & 0.11 \\
\hline $\mathrm{MgO}$ & 0.21 & 0.21 & 0.25 & 0.20 & 0.22 & 0.25 & 0.23 & 0.23 & 0.22 & 0.24 \\
\hline $\mathrm{CaO}$ & 1.52 & 1.82 & 2.14 & 1.61 & 1.59 & 1.78 & 1.69 & 1.80 & 1.75 & 1.77 \\
\hline $\mathrm{Na}_{2} \mathrm{O}$ & 3.46 & 3.18 & 3.54 & 1.19 & 3.37 & 3.56 & 3.40 & 3.40 & 3.47 & 3.46 \\
\hline $\mathrm{K}_{2} \mathrm{O}$ & 2.89 & 2.57 & 2.55 & 1.90 & 2.66 & 2.78 & 2.83 & 2.68 & 2.69 & 2.65 \\
\hline $\mathrm{P}_{2} \mathrm{O}_{5}$ & 0.00 & 0.12 & 0.00 & 0.00 & 0.06 & 0.00 & 0.00 & 0.00 & 0.00 & 0.03 \\
\hline Total & 95.56 & 95.74 & 98.33 & 96.10 & 92.05 & 95.27 & 96.05 & 95.76 & 96.30 & 96.78 \\
\hline
\end{tabular}

ic arc systems showing moderate $\mathrm{K}$ enrichment, and contrast with clinopyroxene grains crystallized from a low-K magma, which tend to show somewhat lower proportion of $\mathrm{Ca}$ at similar Fe values (Ewart, 1979, 1982; Gill, 1981).

Orthopyroxene grains are iron-rich enstatites with analyses falling largely in the hypersthene compositional field. In Sample 156-949A$1 \mathrm{H}-1,75-77 \mathrm{~cm}$, analyses extend to the boundary with the ferrosilite field $\left(\mathrm{Fs}_{50}\right)$. One analysis from Sample 156-949A-1H-CC, 5-7 cm, contains a relatively high $\mathrm{Mg} / \mathrm{Fe}$ value and falls in the bronzite field. Samples 156-949A-1H-1, 35-37 cm, and 156-949A-1H-1, 75-77 $\mathrm{cm}$, show a restricted range of $\mathrm{Mg} / \mathrm{Fe}$ values with Fs values of $42-50$ $\mathrm{mol} \%$, whereas orthopyroxene grains in Sample 156-949A-1H-CC, 5-7 $\mathrm{cm}$ range between $\mathrm{Fs}_{22}$ and $\mathrm{Fs}_{49}$ mol\%. The Fe content of the orthopyroxenes is higher than that of the clinopyroxenes from the same samples (Fig. 4). Although the fragmental nature of the samples prevents establishment of co-existing mineral assemblages, this is con- sistent with the observation of Gill (1981) that orthopyroxene phenocrysts have more variable and higher $\mathrm{Fe} / \mathrm{Mg}$ values than coexisting augite grains. The calcium content of the orthopyroxenes is around $1-3 \mathrm{~mol} \%$.

\section{Amphibole}

Amphibole compositions were determined from Samples 156949A-1H-1, 75-77 cm, 156-949A-1H-CC, 5-7 cm, and 156-949B19X-4, 10-11 cm (Table 5). All are calcic amphiboles with $(\mathrm{Ca}+$ $\mathrm{Na})_{B}>1.34$ and $\mathrm{Na}_{B}<0.67$ and belong to the hornblende series. In terms of the classification scheme of Leake (1978), analyses fall in the magnesio-hornblende field with $(\mathrm{Na}+\mathrm{K})_{\mathrm{A}}<0.50$, $\mathrm{Ti}<0.50$, $\mathrm{Mg} /(\mathrm{Mg}+\mathrm{Fe})>0.50$, and $\mathrm{Si}$ between 6.50 and 7.25. All analyses show a restricted range of $\mathrm{Mg}: \mathrm{Fe}$ ratios (Fig. 5) with Sample 156949A-1H-1, 75-77 cm, having Fe values of 30-36 mol\% and Sam- 

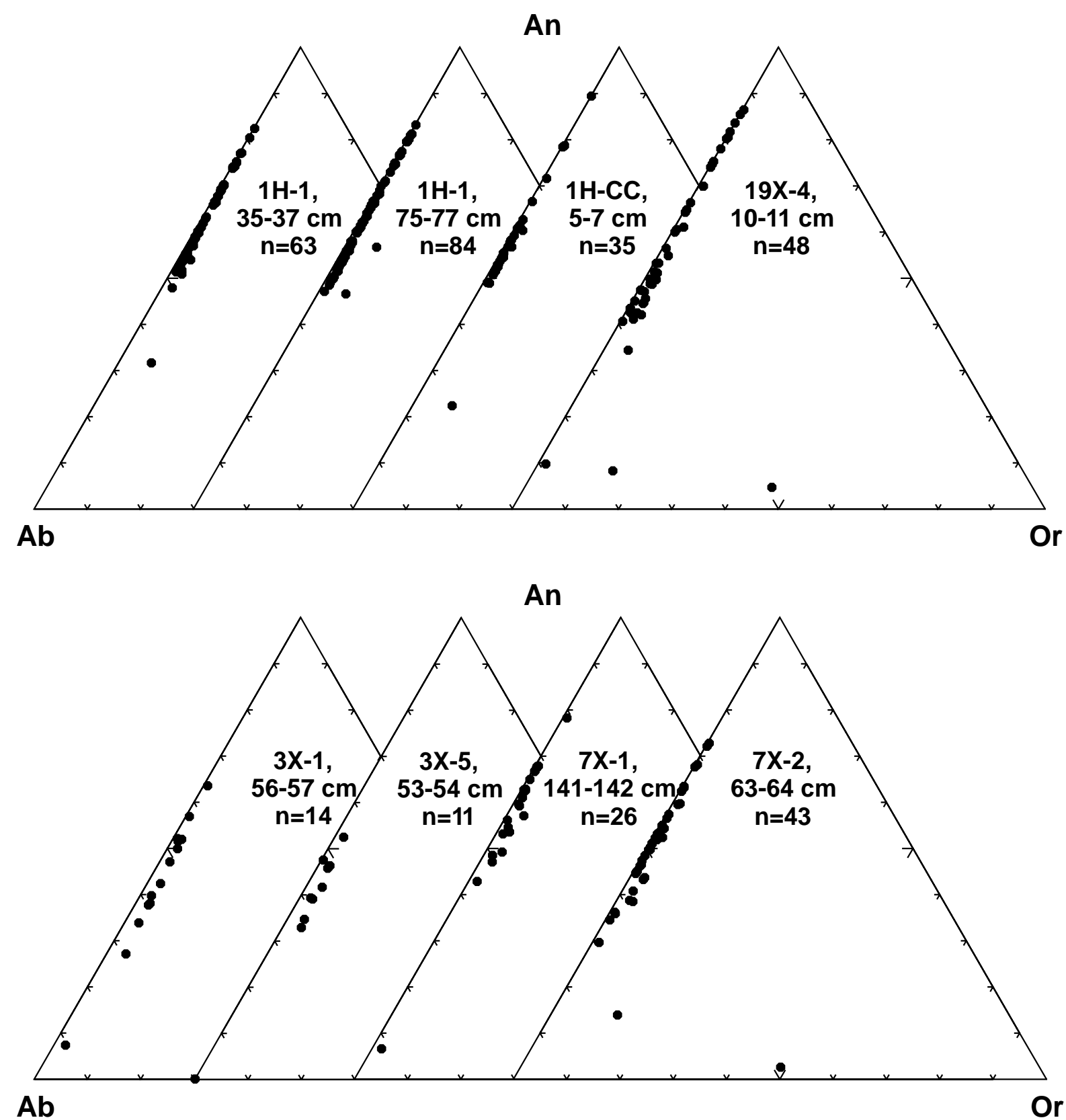

Figure 2. Composition of feldspar grains in volcanic ash samples from Site 949. $n=$ number of analyses.

ples 156-949A-1H-CC, 5-7 cm, and 156-949B-19X-4, 10-11 cm, falling in the narrow compositional range between 27 and $28 \mathrm{~mol} \%$ $\mathrm{Fe}$. The higher Mg:Fe ratio of Sample 156-949A-1H-CC, 5-7 cm, relative to $156-949 \mathrm{~A}-1 \mathrm{H}-1,75-77 \mathrm{~cm}$, is consistent with relations observed in orthopyroxene compositions. Amphibole analyses from these two samples, (excluding outlier compositions in Sample 156949A-1H-CC, 5-7 cm), lie along the clinopyroxene-orthopyroxene tie line in terms of Wo-En-Fs (Fig. 5). Although, as with the pyroxene data, it is not possible to determine primary coexisting mineral assemblages, the intra-element compositional relationships of the ferromagnesian phases are consistent with their being in equilibrium and having been derived from the same magma source.

$\mathrm{Na}+\mathrm{K}$ and $\mathrm{Al}^{\text {iv }}$ contents of the amphiboles are relatively low at around 0.4 and $1 \mathrm{~mol} \%$ respectively, and they are characterized by relatively high $\mathrm{Si}$ to $\mathrm{Ca}$ plus alkalis and low $\mathrm{Al}$ relative to $\mathrm{Mg}+\mathrm{Fe}+$
$\mathrm{Mn}$. These characteristics are those of continental margin rather than intra-oceanic island-arc hornblendes, and indicate that the source magma was likely of high silica composition and had interacted with relatively thick crust (Jakes and White, 1972; Ewart, 1979).

\section{Volcanic Glass}

Volcanic glass compositions were determined for Samples 156949A-1H-1, 35-37 cm, 156-949A-1H-1, 75-77 cm, 156-949A-1HCC, 5-7 cm, and 156-949B-19X-4, 10-11 cm (Table 6). Analytical totals are generally low, falling between $91.1 \%$ and $98.3 \%$. This suggests that, although the glass appears optically "fresh," it has undergone some degree of alteration to which it is particularly susceptible because of its amorphous character and high surface area to volume ratio. The analyses preserve inter-element variation characteristic of 


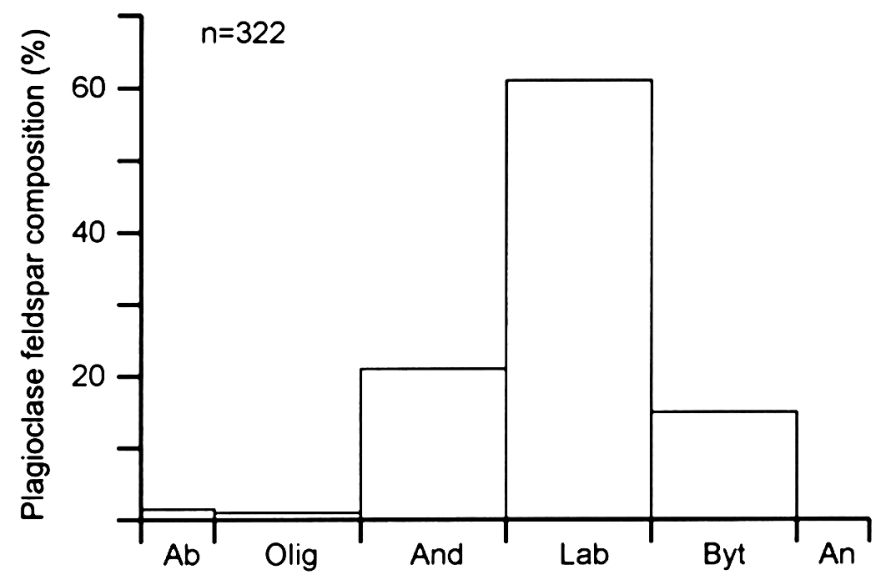

Figure 3. Histogram of plagioclase feldspar compositions from Site 949 volcanic ash samples. Ab $=$ albite, Olig $=$ oligoclase, And $=$ andesine, Lab $=$ labradorite, $\mathrm{Byt}=$ bytownite, and $\mathrm{An}=$ anorthite. $\mathrm{n}=$ number of analyses .
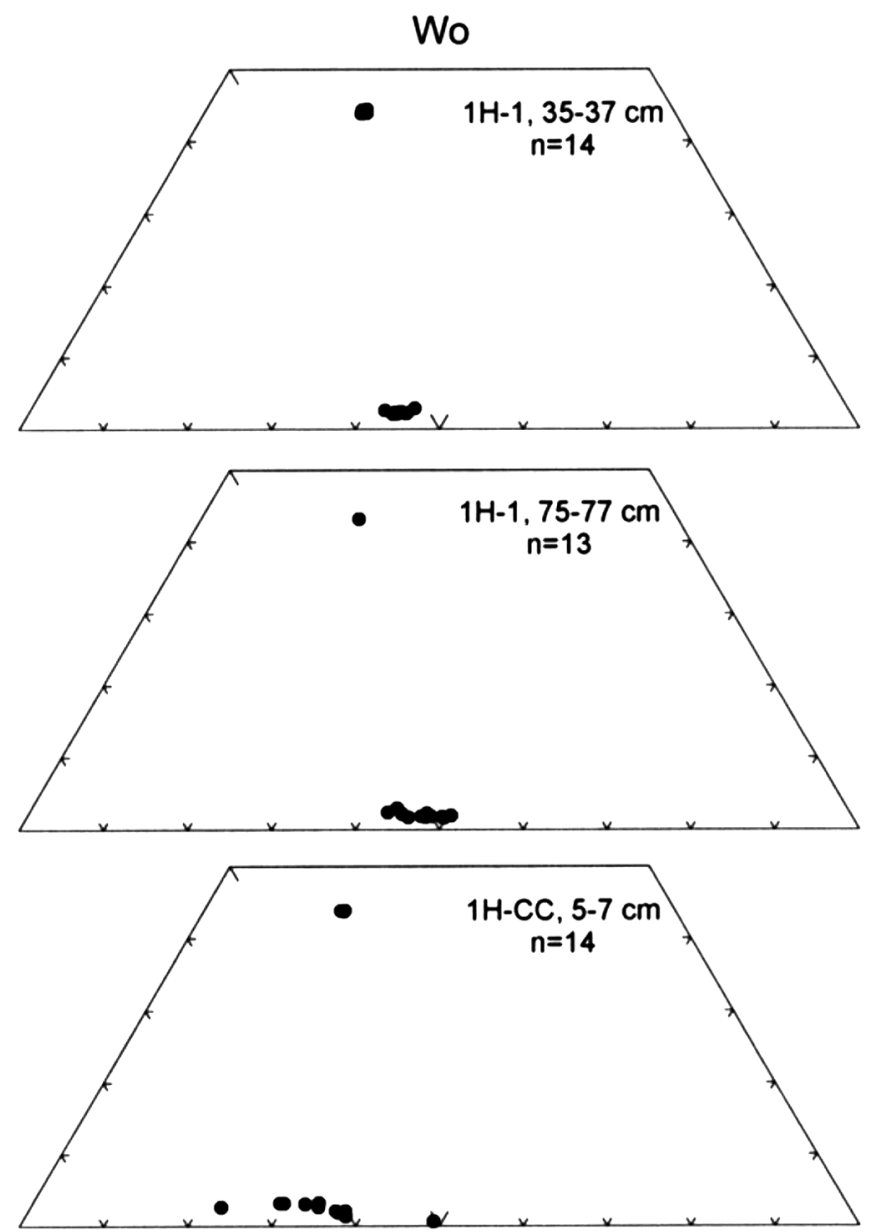

En

Fs

Figure 4. Wo-Fs-En plot of pyroxene grains from Site 949 volcanic ash samples. $\mathrm{n}=$ number of analyses.

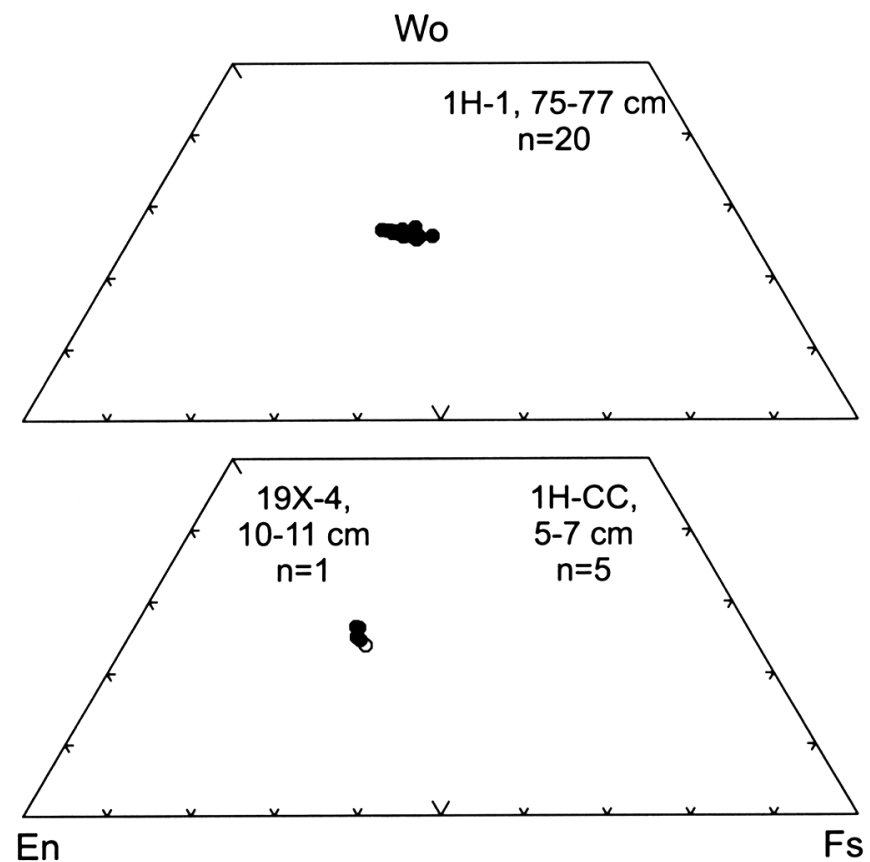

Figure 5. Wo-Fs-En plot of amphibole grains from Site 949 volcanic ash samples. In lower diagram, the open circle is an analysis from Sample 156949B-19X-4, 10-11 cm, and the filled circles are analyses from Sample 156$949 \mathrm{~A}-1 \mathrm{H}-\mathrm{CC}, 5-7 \mathrm{~cm} . \mathrm{n}=$ number of analyses.

igneous rocks (Figs. 6, 7) and alteration was probably mainly simple hydration as has been demonstrated by earlier studies of deep-sea tephra (e.g., Cadet et al., 1981, 1982; Pouclet et al., 1985, 1986, 1990).

$\mathrm{SiO}_{2}$ ranges from $65 \%$ to $78 \%$, covering the compositional range of dacite and rhyolite. Individual samples show a more restricted range: Sample 156-949B-19X-4, 10-11 cm, 65\%-71\%; 156-949A$1 \mathrm{H}-1,75-77 \mathrm{~cm}, 68 \%-78 \%$; 156-949A-1H-1, 35-37 cm, 69\%-75\%; and $156-949 \mathrm{~A}-1 \mathrm{H}-\mathrm{CC}, 5-7 \mathrm{~cm}, 70 \%-75 \%$. These data add weight to the conclusion of Sigurdsson and Carey (1981) that rhyodacites are the dominant products of volcanism in the Lesser Antilles, despite the mainly andesitic composition of igneous rocks exposed in the arc (Bouysse et al., 1990). This is partly accounted for by the highly explosive mode of eruption of silicic magmas and resultant wide distribution of silicic tephra, compared with the generally less violent pyroclastic eruption, and hence more local distribution, of the products of more mafic magmas. It is also contributed to by the more silicic composition of volcanic glass compared with bulk rock compositions.

Figures 6 and 7 outline trends of selected major elements with respect to $\mathrm{SiO}_{2}$. Sample 156-949B-19X-4, 10-11 cm, shows a pronounced decrease in $\mathrm{FeO}, \mathrm{MgO}$, and $\mathrm{CaO}$, with increasing $\mathrm{SiO}_{2}$ and constant to slightly increasing $\mathrm{Na}_{2} \mathrm{O}$ and $\mathrm{K}_{2} \mathrm{O}$. Other samples, in contrast, show constant values of $\mathrm{FeO}$ and $\mathrm{MgO}$ with increasing $\mathrm{SiO}_{2}$, although with some scatter, and no clear trend in $\mathrm{Na}_{2} \mathrm{O}$ and $\mathrm{K}_{2} \mathrm{O}$ with change in $\mathrm{SiO}_{2}$ content. Samples 156-949A-1H-1, 75-77 cm, and 156-949A-1H-CC, 5-7 cm, show considerable scatter of individual analyses on these plots, suggesting derivation of these tephra from more than one magma source, whereas the restricted compositional range of glass (and pyroxene) argues against any mixing of magma sources for Sample 156-949A-1H-1, 35-37 cm.

Sample 156-949B-19X-4, 10-11 cm, is characterized by uniformly low $\mathrm{K}_{2} \mathrm{O}$ values $(<1 \%)$, indicative of derivation from a low-K magma (Fig. 7). $\mathrm{K}_{2} \mathrm{O}$ values of Sample 156-949A-1H-CC, 5-7 cm, range from $1.2 \%$ to $2.7 \%$ and extend from the low- $\mathrm{K}$ into the medium-K 
field on the $\mathrm{K}_{2} \mathrm{O}$ vs. $\mathrm{SiO}_{2}$ classification diagram. Analyses from Sample 156-949A-1H-1, 75-77 cm, are divisible into two groups on the basis of $\mathrm{K}_{2} \mathrm{O}$ content. One group, consisting of only three analyses, is characterized by $\mathrm{K}_{2} \mathrm{O}$ contents of $1.7 \%-1.9 \%$ and plots in the lower part of the medium-K field. A second group, containing the bulk of the analyses, has $\mathrm{K}_{2} \mathrm{O}$ values of $2.4 \%-2.9 \%$. Glass from Sample 156949A-1H-1, 35-37 cm, is characterized by a uniformly high $\mathrm{K}_{2} \mathrm{O}$ content of around $2.6 \%-2.8 \%$ and plots in the medium-K field. On the AFM diagram (Fig. 8), the glass analyses show similar compositional trends, but with all analyses from Sample 156-949B-19X-4, $10-11 \mathrm{~cm}$, plotting in the tholeiitic field of Irvine and Baragar (1971), and the other samples falling in their calc-alkaline field. The tholeiitic character of Sample 156-949B-19X-4, 10-11 cm, is noteworthy for it is of similar age to early Miocene tholeiitic rocks exposed on the island of Martinique, close to Site 949 (Fig. 1). Later Miocene and Pleistocene volcanic rocks from Martinique, and from Site 949, are of medium-K calc-alkaline composition (Bouysse et al., 1990; Maury et al., 1990).

\section{ACKNOWLEDGMENTS}

This report was improved by the comments of Peter Blum, Herve Cambray, James Allan, and an anonymous reviewer. We appreciate the help of Norm Pearson and Carol Lawson with microprobe analyses, and Brian Cikara in sample preparation. The research was supported by the Australian Research Council.

\section{REFERENCES}

Bednarz, U., and Schmincke, H-U., 1994. Composition and origin of volcaniclastic sediments in the Lau Basin (Southwest Pacific), Leg 135 (Sites 834-839). In Hawkins, J.W., Parsons, L.M., Allan, J., Proc. ODP, Sci. Results, 135: College Station, TX (Ocean Drilling Program), 51-74.

Bouysse, P., Westercamp, D., and Andreieff, P., 1990. The Lesser Antilles island arc. In Moore, J.C., Mascle, A., et al., 1990. Proc. ODP, Sci. Results, 110: College Station, TX (Ocean Drilling Program), 29-44.

Cadet, J.-P., Thisse, Y., Pouclet, A., Bardintzeff, J.-M., and Stephan, J.-F., 1981. Tephra from Deep Sea Drilling Project Leg 66: Middle America Trench transect (Southern Mexico). In Watkins, J.S., Moore, J.C., et al., Init. Repts. DSDP, 66: Washington (U.S. Govt. Printing Office), 687-698.

Cadet, J.-P., Pouclet, A., Thisse, Y., Bardintzeff, J.-M., and Azema, J., 1982. Middle America Neogene explosive volcanism and ash layers: evidence from the Middle America Trench transect. In Aubouin, J., von Huene, R., et al., Init. Repts. DSDP, 67: Washington (U.S. Govt. Printing Office), $475-491$.

Carey, S.N., and Sigurdsson, H., 1980. The Rouseau Ash: deep-sea tephra deposits from a major eruption on Dominica, Lesser Antilles Arc. J. Volcanol. Geotherm. Res., 7: 67-86.

Ewart, A., 1979. A review of the mineralogy and chemistry of TertiaryRecent dacitic, lattice, rhyolitic, and related silicic volcanic rocks. In Barker, F., (Ed.), Trondhjemites, dacites, and related rocks. Developments in petrology, 6: 13-121.

1982. The mineralogy and petrology of Tertiary-Recent orogenic volcanic rocks with special reference to the andesite-basalt compositional range. In Thorpe, R.S., (Ed.), Andesites: New York (John Wiley).
Fisher, R.V. and Schmincke, H.-U., 1984. Pyroclastic rocks: New York (Springer Verlag).

Gill, J.B., 1981. Orogenic andesites and plate tectonics: Berlin (Springer Verlag).

Irvine T.N., and Baragar W.R., 1971. A guide to the chemical classification of common igneous rocks. Can. J. Earth Sci., 8: 523-548.

Jakes, P., and White, A.J.R., 1972. Hornblendes from calc-alkaline volcanic rocks of island arcs and continental margins. Amer. Mineral., 57: 887902.

Leake, B.E., 1978. Nomenclature of amphiboles. Can. Mineral., 16: 501-520.

LeMaitre, R.W., 1989. A classification and glossary of igneous rocks and glossary of terms: Oxford (Blackwell).

Maury, R.C., Westbrook, G.K., Baker, P.E., Bouysse, P., and Westercamp, D., 1990. Geology of the Lesser Antilles. In Dengo, G., and Case, J.E., (Eds.), The Caribbean region. The geology of North America: Boulder (Geological Society of America).

Natland, J.H., 1984. Occurrences of air-fall volcanic ash derived from the Lesser Antilles Arc at Leg 78A Drill Sites. In Biju-Duval, B., Moore, J.C., et al., Init. Repts. DSDP, 78A: Washington (U.S. Govt. Printing Office), 369-375.

Nielsen, C.H., and Sigurdsson, H., 1981. Quantitative methods for electron microprobe analysis of sodium in natural and synthetic glasses. Amer. Mineral., 66: 547-552.

Peccerillo, and Taylor, 1976. Geochemistry of Eocene calc-alkaline volcanic rocks from Kastamonu area, northern Turkey. Contrib. Mineral. Petrol., 58: 63-81.

Pouchou, J.L., and Pichoir, F., 1984. A new model for quantitative X-ray microanalysis of homogeneous samples. Recherche Aerospatiale, 5: 13-38.

Pouclet, A., Cadet, J.-P., Fujioka, K., and Bourgois, J., 1985. Ash layers from Deep Sea Drilling Project Leg 84: Middle America Trench transect. In von Huene, R., Aubouin, J., et al., Init. Repts. DSDP, 84: Washington (U. S. Govt. Printing Office), 481-489.

Pouclet, A., Cambray, H., Cadet, J.-P., Bourgois, J., and De Weaver, P., 1990. Volcanic ash from Leg 112 off Peru. In Suess, E., von Huene, R., et al., Proc. ODP, Sci. Results, 112: College Station, TX (Ocean Drilling Program), 465-480.

Pouclet, A., Fujioka, K., Charvet, J., and Cadet, J.-P., 1986. Petrography and geochemistry of volcanic ash layers from Leg 87A, Nankai Trough (South Japan). In Kagami, H., Karig, D. E., and Coulbourn, W.T., et al., Init. Repts. DSDP, 87A: Washington (U.S. Govt. Printing Office), 695-701.

Shipley, T.H., Ogawa, Y., Blum, P., et al., 1995. Proc. ODP Init. Repts., 156: College Station, TX (Ocean Drilling Program).

Sigurdsson, H., and Carey, S.N., 1981. Marine tephrochronology and Quaternary explosive volcanism in the Lesser Antilles arc. In Self, S., and Sparks, R.S.J., Tephra Studies: Dordrecht, Holland (D. Reidel), 255-280.

Sigurdsson, H., Sparks, R.S.J., Carey, S.N., and Huang, T.C., 1980. Volcanogenic sedimentation in the Lesser Antilles arc. J. Geology, 88: 523-540.

Date of initial receipt: 5 February 1996

Date of acceptance: 1 October 1996

Ms 156SR-014 

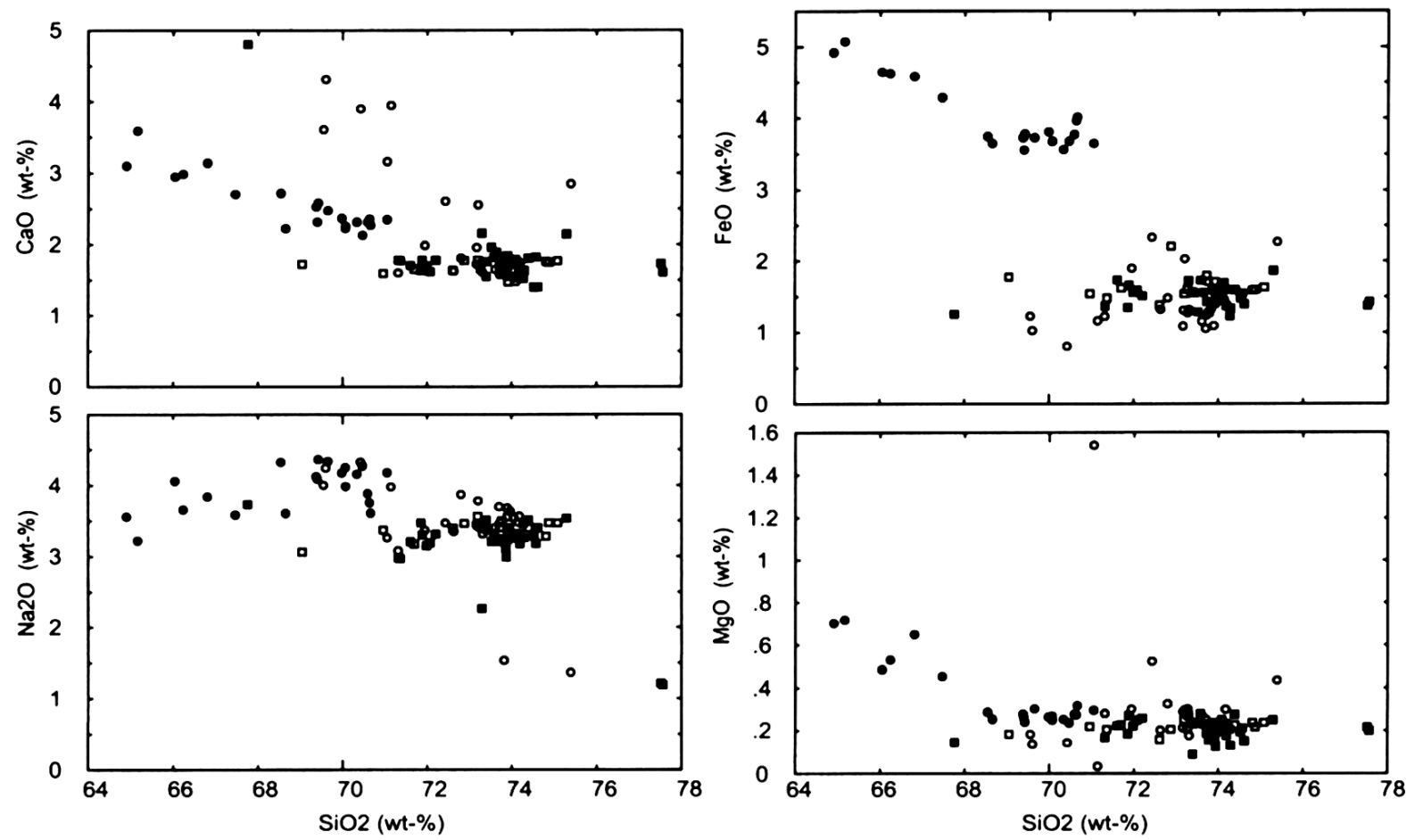

Figure 6. Variation diagrams of selected major elements with respect to $\mathrm{SiO}_{2}$ for glass analyses from Site 949 volcanic ash samples. Open squares $=\mathrm{Sample}$ 156-949A-1H-1, 35-37 cm; solid squares = Sample 156-949A-1H-1, 75-77 cm; open circles $=$ Sample 156-949A-1H-CC, 5-7 cm; and solid circles $=$ Sample 156-949B-19X-4, 10-11 cm.
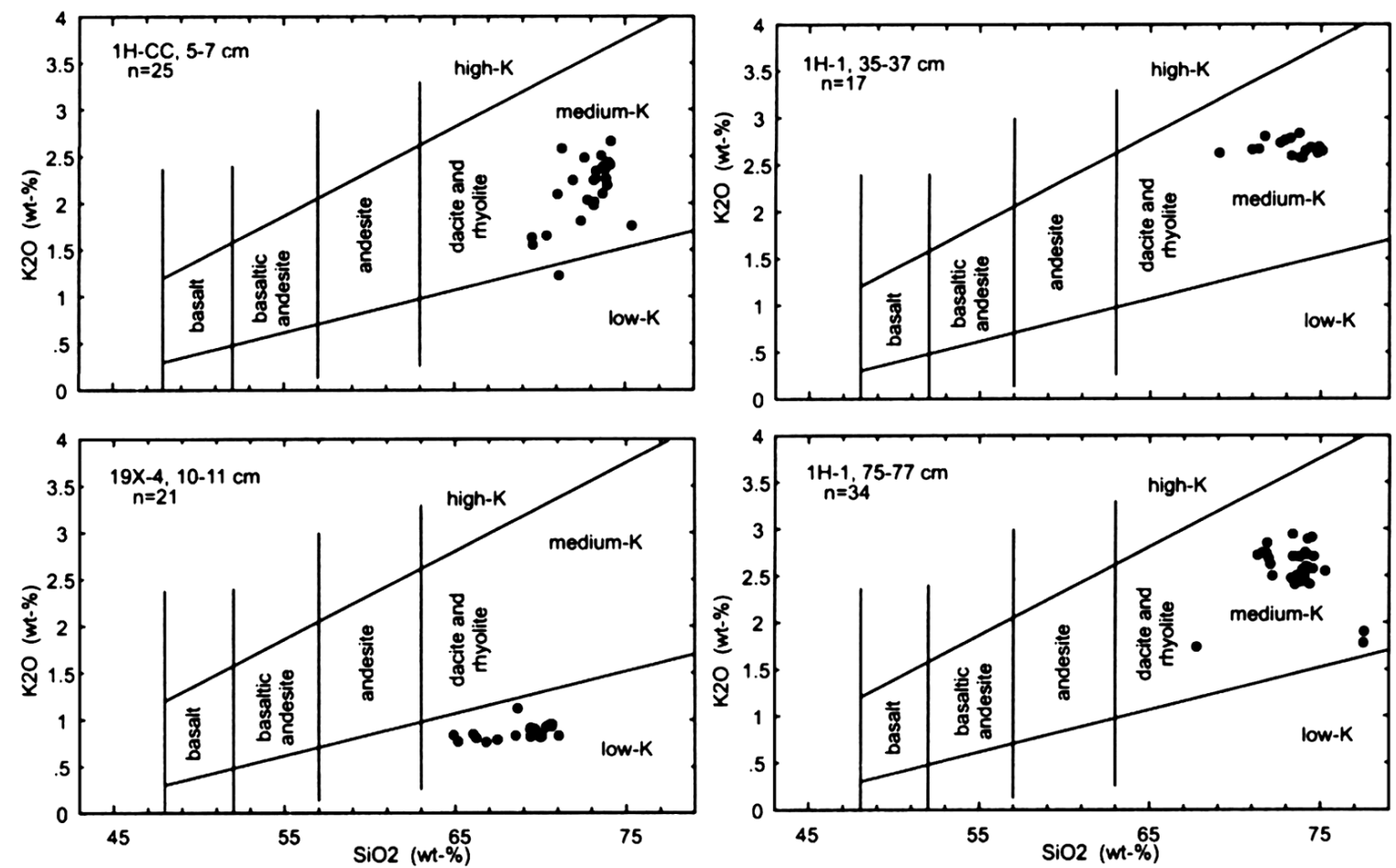

Figure 7. Plot of $\mathrm{K}_{2} \mathrm{O}$ vs. $\mathrm{SiO}_{2}$ for glass from Site 949 volcanic ash samples. Fields are those defined by Peccerillo and Taylor (1976) as modified by Le Maitre (1989). $\mathrm{n}=$ number of analyses. 

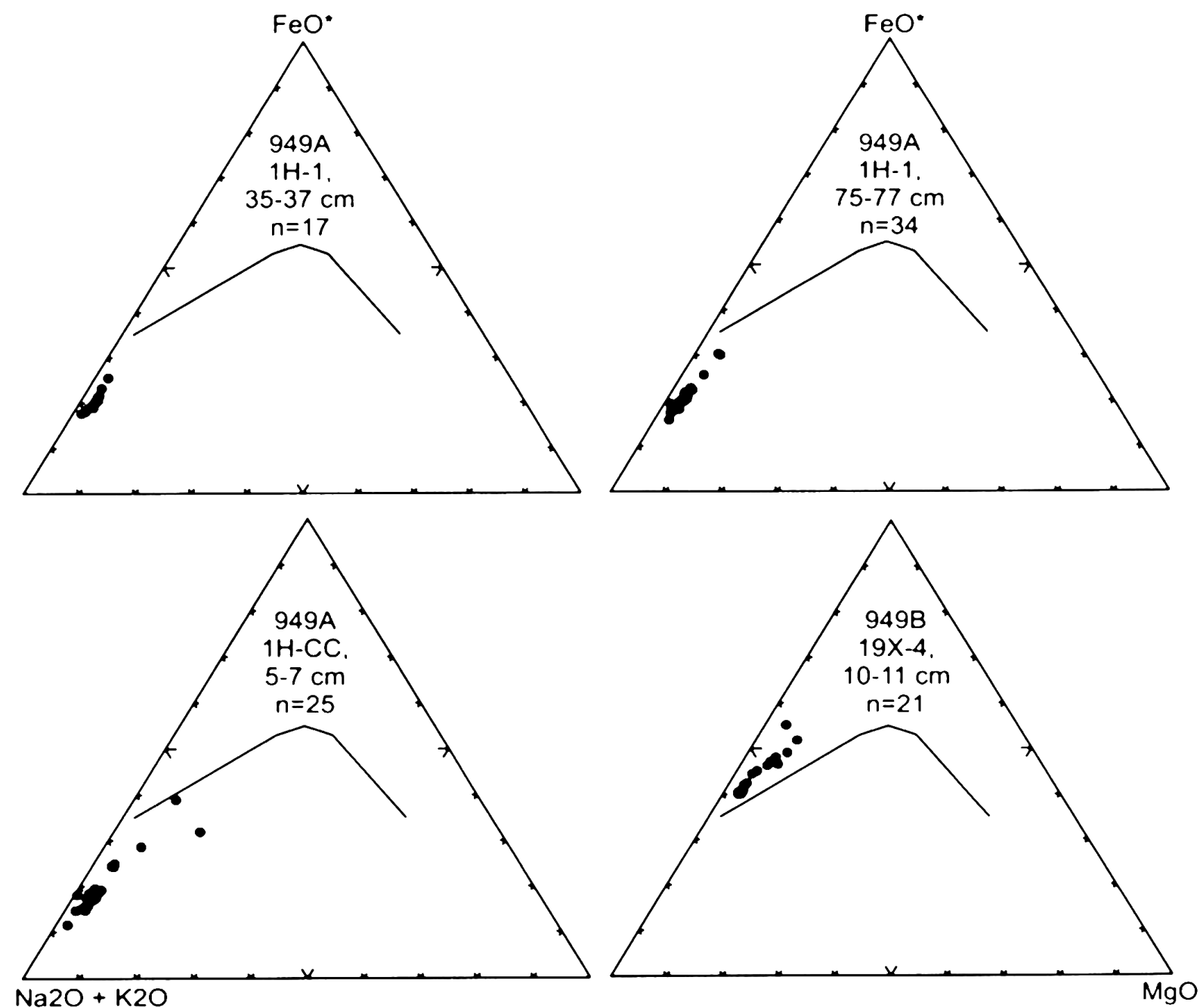

Figure 8. AFM ( $\mathrm{Na}+\mathrm{K}: \mathrm{FeO} * \mathrm{MgO})$ plot of glass from Site 949 volcanic ash samples. Line separates tholeiitic field (above) from calc-alkaline field (below) after Irvine and Baragar (1971). $\mathrm{n}=$ number of analyses. $\mathrm{FeO}^{*}=$ total iron expressed as $\mathrm{FeO}$. 